\title{
Mecanismos de financiación y gestión de recursos financieros del sector de la construcción, infraestructura y megaproyectos en Colombia
}

\author{
Jahir A. Gutiérrez O.* \\ Juan D. Vega** \\ Santiago Osorio A.***
}

Fecha de recibido: 22 de octubre de 2017

Fecha de aprobado: 15 de junio de 2018

Para citar: Gutiérrez, J. A., Vega, J. D., \& Osorio, S. (2019). Mecanismos de financiación y gestión de recursos financieros del sector de la construcción, infraestructura y megaproyectos en Colombia. Universidad \& Empresa, 21(36), 119-148. DOI: http://dx.doi.org/10.12804/revistas.urosario.edu.co/empresa/a.6191

* Economista de la Universidad de Medellín, Colombia. Ph.D. en Administración Pública, Atlantic International University (AIU), Honolulú, Estados Unidos. Magíster en Desarrollo, Universidad Pontificia Bolivariana, Medellín, Colombia. Estudios en Sistemas de Información Geográfica, Universidad San Buenaventura (USB), Medellín, Colombia. Investigador del Grupo de Investigación Pluriverso en Postgrados, y coordinador y docente investigador del grupo de investigación Gestión empresarial, Facultad de Ciencias administrativas y económicas, Universidad CES. Correo electrónico: jagogutierrez@gmail.com

** Economista Industrial de la Universidad de Medellín, Colombia. Especializado en Alta Gerencia con Énfasis en Calidad de la Universidad de Antioquia, Medellín, Colombia. Especializado en Asesoría y Consultoría de la Universidad de Antioquia, Medellín, Colombia, y en Gerencia de Proyectos de la Universidad del Tolima, Ibagué, Colombia. Magíster en Administración de Negocios con Énfasis en Negocios Internacionales de la Universidad EAFIT, Medellín, Colombia. Ph.D en Management de la Universidad de Siracusa, Estados Unidos. Docente, investigador del Grupo de Investigación en Gestión Empresarial de la Universidad CES. Coordinador de proyectos Facultad Ciencias administrativas y económicas Universidad CES. Correo electrónico: jvega@ces.edu.co

*** Estudiante de Administración de empresas de sexto semestre, Universidad CES. Integrante del Grupo de investigación Gestión empresarial, línea de Estrategia, dirección y reputación corporativa. Correo electrónico: santiosorioa@hotmail.com 


\section{Resumen}

El escrito plantea la consecuencia que tienen los mecanismos de financiación y de gestión de recursos financieros con respecto al tamaño de las obras de construcción, infraestructura y megaproyectos en Colombia. La tendencia a medir la relación financiera y los costos que dichas intervenciones exigen es tan desconocida, como la propia consideración de los impactos luego de la terminación de las obras. El análisis exploratorio y descriptivo sirve de sustento básico para ampliar las razones por las cuales se cree que el propio tamaño de las obras reduce el análisis académico e investigativo sobre ello. El análisis financiero de las grandes obras no termina con la disposición de ellas, al contrario, implica la internalización de ellas en donde son asentadas.

Palabras clave: megaproyectos; toma de decisiones; riesgo financiero, Infraestructuras; Mercados financieros.

\section{Finance Mechanisms and Management of Financial Resources of Construction, Infrastructure and Megaprojects in Colombia Abstract}

The brief raises the consequence that the mechanisms of financing and management of financial resources should have with respect to the size of the works of construction, infrastructure and mega-projects in Colombia. The tendency to measure the financial relationship and the costs of such interventions require is as unknown as the own consideration of impacts after the completion of the works do. The exploratory and descriptive analysis serves as basic sustenance to expand the reasons why it is believed that the sheer size of the works reduces the academic and research analysis on it. The financial analysis of the works does not end with the disposal of them, on the contrary, it involves the internalization of them where they are seated.

Keywords: Mega-projects, decision making, financial risk, infrastructure; financial markets.

\section{Mecanismos de Financiamento e Gestão de Recursos Financeiros do Setor da Construção, Infraestrutura e Megaprojetos na Colômbia Resumo}

O escrito apresenta a consequência que devem ter os mecanismos de financiamento e de gestão de recursos financeiros com respeito ao tamanho das obras de construção, infraestrutura e Megaprojetos na Colômbia. A tendência a medir a relação financeira e os custos que sitas intervenções exigem, [e tão desconhecida, quanto a própria consideração dos impactos, logo da terminação das obras. A análise exploratória e descritiva, serve de sustento básico para ampliar os motivos pelos quais acha-se que o próprio tamanho das obras reduze a análise acadêmica e investigativa sobre isso. A análise financeira das grandes obras não termina com a disposição delas, ao contrário, implica a internalização delas onde são assentadas.

Palavras-chave: megaprojetos, tomada de decisões, risco financeiro, Infraestruturas, Mercados financeiros. 


\section{Introducción}

Es poco lo que se advierte en materia del componente económico y financiero que cubren las obras de construcción, infraestructura y megaproyectos, hecho que refleja la invisibilidad de la gestión que debe hacerse para obtener recursos que apoyen dichas intervenciones. En este sentido, si bien las grandes obras comprometen conocimientos propios, es menester ubicar en igual dirección los elementos que atañen a las finanzas. También es necesario reflexionar sobre la realidad de la estructura financiera para solventar las necesidades de dichas actividades, y si efectivamente, la disposición financiera se ajusta a las obras.

El tamaño de las obras parece complejizar aún más la correlación entre demandas y ofertas financieras de quienes a través de consorcios o empresas de infraestructura optan por mejorar u optimizar sus condiciones financieras, a través del apalancamiento en créditos para la ejecución de las intervenciones. Es de indicar que las evaluaciones y mediciones que surgen al respecto, generalmente aparecen cuando los proyectos terminan desfinanciados o no alcanzan a completar las ejecuciones, por desconocer o pasar por alto requerimientos complementarios, de diseño o de proyección de las intervenciones.

La evidencia muestra que la participación sobre los proyectos de infraestructura corre por cuenta de las grandes agencias o consorcios internacionales o de la banca multilateral, que funge en calidad de prestamista de primera mano para estos proyectos, aunque la fuente idónea de recursos para estos menesteres debería proceder de las entidades financieras bancarias de corte nacional e internacional. Así que, el acompañamiento y presencia de la banca crediticia y financiera en Colombia está dirigida a proyectos muy puntuales, que no revisten las grandes apuestas esperadas sobre el sector.

Es importante establecer las perspectivas que ofrece el campo de las obras de construcción, infraestructura y megaproyectos para las entidades bancarias, crediticias y financieras del país, y como ellas, al entender la magnitud de lo que está en juego, pueden ordenar sus portafolios hacia frentes más que consolidados en las intervenciones físicas, no solo por la importante disposición de recursos del gobierno desde el orden central para acometerlos. Además, la composición de carácter internacional y local de los mismos exige un replanteamiento del sector financiero frente a la manera como concibe a este. 
La exploración amplia de lo que implica la revisión de los pormenores financieros de las obras destaca de nuevo el interés por orientar la evaluación de proyectos y otros menesteres hacia dichos fines. A su vez, la cuantificación y valoración de los detalles que allí emanan sirven para estimar la conveniencia de considerar el componente financiero de los proyectos más importantes que se vienen considerando en el país, y que de paso obligan a los entes territoriales a rearmar sus estructuras financieras, por lo que implica para ellas, el administrar, costear o beneficiarse de los impactos generados.

La estructura del artículo responde a los siguientes componentes de análisis: En primera instancia, de una revisión sobre las condiciones bancarias, crediticias y financieras que se tienen para la inyección en obras de infraestructura. En segundo lugar, la participación de diferentes estamentos, instituciones y organismos internacionales insertos en financiar dichos proyectos, y por último, evidenciar las perspectivas que se tienen frente a la banca y las entidades financieras en Colombia, en cuanto a su capacidad y condiciones para ser considerados músculos de recursos para estos proyectos.

\section{Justificación}

La problemática identificada que dio lugar a este producto investigativo tiene su inicio en una necesidad que se ha evidenciado en la industria de la construcción, la cual va directamente relacionada con los requerimientos de recursos financieros por parte de las constructoras, no solo en la ejecución del presupuesto proyectado por estas, sino en los diferentes factores que surgen en medio de la realización de sus actividades. Entre dichos factores se encuentran inconsistencias de cumplimiento por falta presupuestal, reprocesos innecesarios en la planeación que aumentan el costo, recursos no previstos y obtenidos, entre otras variables monetarias, que obligan a las empresas participantes del sector a recurrir a intermediarios financieros internacionales como proveedores de portafolios crediticios, reduciendo consecuentemente la participación de la banca colombiana en la prestación de estos productos, anexado a la falta de fortaleza que brinda la banca colombiana en dar una oferta específica al sector.

Dado lo anterior, la investigación gira entorno a la reflexión demostrativa sobre cómo la banca colombiana cuenta con la capacidad de competir en el mercado frente 
a otras entidades financieras internacionales en materia referida a los créditos de construcción, partiendo de la posición de que los bancos colombianos pueden expandir sus portafolios crediticios. La expansión del portafolio es clave para participar como oferente que supla necesidades del sector, el cual tiene aspectos de ganancias que minimizan el riesgo a los mismos entes financieros (tomando como ejemplo la demanda gubernamental en el desarrollo de construcción, lo cual requiere intermediarios financieros adaptables al entorno demandado). En este escenario, es factible el estudio investigativo que aporte de manera conceptual aplicativa al sector, permitiendo así el análisis de las posibilidades que existen de trabajar a modo de joint venture tanto para la parte constructora como para la financiera, con el objetivo de aumentar los beneficios de la inversión en este ámbito.

\section{Estructura bancaria, crediticia y financiera de las obras de construcción e infraestructura}

Las obras de ingeniería civil, física y territorial vienen acompañadas por la disposición de recursos particulares, financieros y multilaterales en tanto obedecen a la magnitud del proyecto a realizar, y es más común para este tipo intervenciones la prevalencia de la evaluación, valoración y toma de riesgo a la hora de inyectar los recursos necesarios. Mientras más grande es la composición de la obra, la disposición de recursos por parte del sector privado se vuelve más exigua en comparación con fuentes de otra índole, cuya disposición de recursos debe acompañarse con impactos diversos. Así,

\footnotetext{
Organismos como el Banco Mundial, el Banco Interamericano de Desarrollo y la CEPAL dividen las infraestructuras en tres clases: la primera, incluye la prestación de servicios de interés público o servicios básicos de infraestructura como: energía eléctrica, gas, telecomunicaciones, abastecimiento de agua, saneamiento, alcantarillado, recogida y eliminación de residuos sólidos. La segunda abarca actividades como carreteras, presas, distritos de riego y drenajes. La tercera, está compuesta por actividades como ferrocarriles, transporte urbano e interurbano, vías navegables, puertos y aeropuertos, entre otros. (Amador, 2011, pp. 191-192):
} 
La construcción tradicionalmente es representada por las edificaciones destinadas al acceso y disposición de vivienda, y de las obras complementarias en servicios públicos domiciliarios e instalaciones básicas. En materia de requerimientos financieros, las empresas constructoras evalúan las condiciones de cada proyecto para que estos alcancen un punto óptimo de rendimiento, que sea atractivo tanto para ellas como para las entidades prestamistas de crédito cuando se trata de vivienda de alto costo; para la vivienda de interés prioritario o social, el apalancamiento financiero proviene del Estado (tabla 1).

Tabla 1. Tipos de infraestructura según función y cobertura geográfica

\begin{tabular}{|c|c|c|c|}
\hline Sectores / Tipos & URBANA & INTERURBANA & INTERNACIONAL \\
\hline Transporte & $\begin{array}{l}\text { Red vial, ferrovías } \\
\text { urbanas. }\end{array}$ & $\begin{array}{l}\text { Carreteras, vías férreas, } \\
\text { vías navegables, puertos, } \\
\text { aeropuertos. }\end{array}$ & $\begin{array}{l}\text { Puertos, aeropuertos, } \\
\text { carreteras, vías navegables, } \\
\text { vías férreas. }\end{array}$ \\
\hline Energía & $\begin{array}{l}\text { Redes de distribución } \\
\text { eléctrica y de gas, plantas } \\
\text { de generación, estaciones } \\
\text { transformadoras. }\end{array}$ & $\begin{array}{l}\text { Redes de transmisión, } \\
\text { gasoductos, oleoductos, } \\
\text { plantas compresoras, } \\
\text { centros de produccióin de } \\
\text { petróleo y gas, centrales } \\
\text { de generación eléctrica. }\end{array}$ & $\begin{array}{l}\text { Redes de transmisión, } \\
\text { gasoductos, oleoductos. }\end{array}$ \\
\hline Telecomunicaciones & $\begin{array}{l}\text { Redes de telefonía } \\
\text { y celular. }\end{array}$ & $\begin{array}{l}\text { Redes de F.O., antenas de } \\
\text { microondas, satélites. }\end{array}$ & $\begin{array}{l}\text { Satélites, cables } \\
\text { submarinos. }\end{array}$ \\
\hline Desarrollo social & Hospitales, escuelas. & $\begin{array}{l}\text { Represas y canales } \\
\text { de irrigación. }\end{array}$ & \\
\hline Medio ambiente & $\begin{array}{l}\text { Parques y reservas } \\
\text { urbanas. }\end{array}$ & $\begin{array}{l}\text { Parques, reservas, territorios } \\
\text { protegidos, circuitos de } \\
\text { ecoturismo. }\end{array}$ & $\begin{array}{l}\text { Parques, reservas, } \\
\text { territorios protegidos, } \\
\text { circuitos de ecoturismo. }\end{array}$ \\
\hline $\begin{array}{l}\text { Información y } \\
\text { conocimiento }\end{array}$ & $\begin{array}{l}\text { Redes, edificios, } \\
\text { TV por cable. }\end{array}$ & $\begin{array}{l}\text { Sistemas de educación } \\
\text { a distancia. }\end{array}$ & Redes. \\
\hline
\end{tabular}

Fuente: Sánchez (2008).

Tradicionalmente, las obras de infraestructura son ejecutadas por empresas constituidas para la creación, ubicación e instalación de proyectos en los que prevalece el interés de promover canales, carreteras y vías de comunicación, así como proyectos que se requieren para instalaciones vinculadas a la actividad productiva puntual. Estas obedecen más a las necesidades que se plantean con respecto a puentes, túneles y vías de acceso, en las que se cuantifican, en el mejor de los casos, los recursos que se requieren a la par con los resultados esperados, en tanto condiciones financieras y técnicas de la obra. Explica Montezuma: 
Según el Banco Interamericano de Desarrollo-BID- "La infraestructura abarca un conjunto de estructuras de ingeniería, equipos e instalaciones de larga vida útil, utilizadas por los sectores productivos y por los hogares". Ésta también puede ser asociada a ese conjunto tanto de elementos como de servicios, que son indispensables para el desarrollo y funcionamiento de procesos sociales, productivos y personales, entre otros. Por tanto, la infraestructura se asimila a un bien de naturaleza pública (2008, p. 17).

Los megaproyectos responden a iniciativas comúnmente de corte estatal, interestatal o multilateral en los que se conjugan, además de los accesos e interconexión, componentes estratégicos que no alcanzan a ser cuantificados eficazmente, debido a la naturaleza e impactos simultáneos que se espera alcanzar con ellos. Normalmente, terminan apalancados por una serie de instituciones y organismos, que a la luz de la planeación estratégica, económica y productiva, esperan que genere condiciones para el crecimiento y el desarrollo. Estos responden a patrones multicriterio de análisis, según lo naliza Livert:

Por ejemplo, y a modo de ilustración, la ventaja del Banco Mundial reside en que puede financiar proyectos de gran magnitud, movilizar una considerable cantidad de recursos de desembolso rápido en situaciones de crisis, y ofrecer conocimientos sobre la base de experiencias en otras regiones del mundo. Por su parte el Banco Interamericano de Desarrollo ha estado, a lo largo de toda su existencia, en estrecho contacto con los países de la región y ha acumulado un profundo conocimiento acerca de su realidad social, institucional y política, lo que le confiere una ventaja en las operaciones destinadas a los sectores sociales, los programas de reformas institucionales (reforma del Estado y de la administración pública) y en temas de gobernabilidad (2011, p. 33).

En Colombia, las referencias que se tienen en cuanto a la dimensión de cada una de las estructuras mencionadas aún es poco observable, sobre todo en relación a los impactos territoriales de las mismas. De allí que la solvencia que estas pueden tener en materia de disposición de recursos para su ejecución sea más dependiente del músculo de capital financiero con el que cuentan, que de la sujeción o articulación que directamente podría tener con el sector bancario y financiero, que para el caso en general, aún no ha hecho una revisión amplia desde lo que podría significar la incursión en dichas apuestas consideradas de alto riesgo (figura 1). 


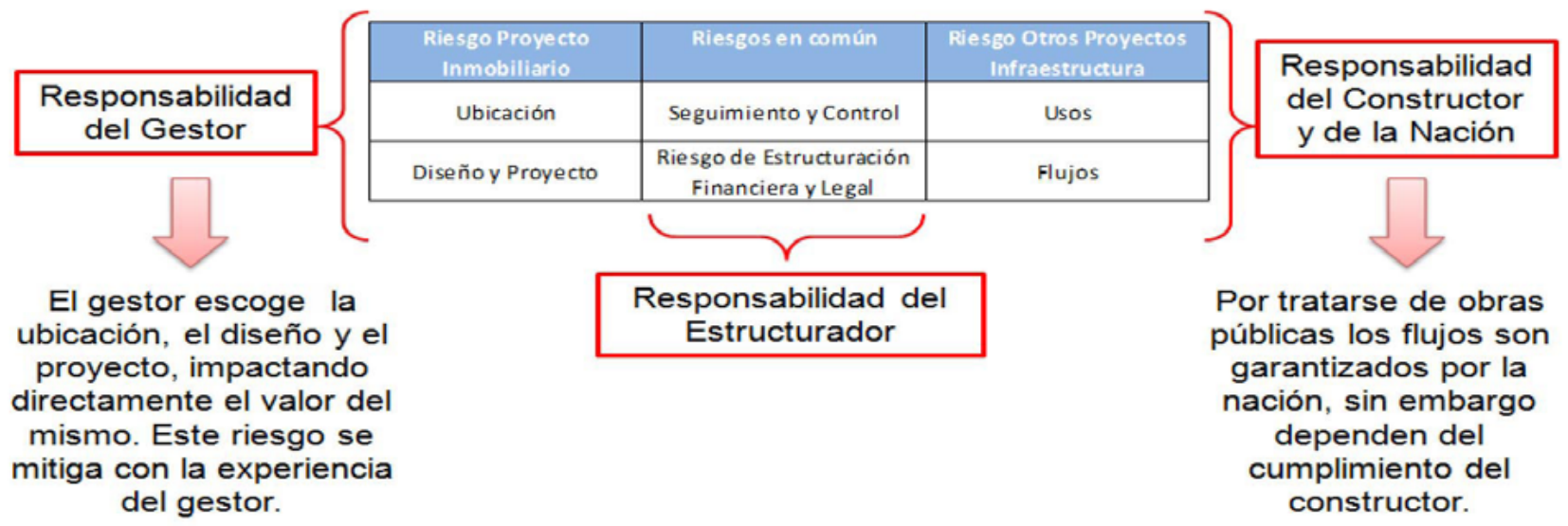

Figura 1. Elementos para la financiación y evaluación de riesgos de los proyectos

Fuente: FIAP (2011).

La construcción destinada a la edificación de obras ha tenido como dirección financiera la disposición que en materia de presupuesto dispone el Estado central. En dicha materia, las líneas estatales consagradas en la Unidad de Poder Adquisitivo (UPAC-1972) y la Unidad de Valor Real (UVR -1998), han constituido en esencia el punto de partida de los recursos estatales para contribuir a la construcción del sector. Estas mismas leyes permitieron la entrada de la banca hipotecaria y crediticia para efectos de la adquisición habitacional, tras la cual muchos colombianos pudieron comprar vivienda. De esta manera,

El endeudamiento bancario ha sido desde hace mucho tiempo la alternativa de financiamiento más accesible para los gobiernos estatales y municipales y, en ocasiones, la única disponible para apoyar la ejecución de proyectos de infraestructura (Villa, 2009, p.17).

El cambio de reformas vendría por cuenta del desajuste presentado entre los pagos vinculados a los créditos hipotecarios, que hasta hace poco estaban sujetos al comportamiento de la tasa de interés, variable que ante cambios financieros bruscos alteraba el abono a capital de los créditos y, por ende, prolongaba aún más la posibilidad de saldar rápidamente dichas cuentas. Así que, en gran medida, la exposición a los movimientos de la tasa de interés provocaría un desajuste tanto en los pagos como en la disposición de créditos. Exponen Fainboim y Rodríguez: 
Los riesgos de los proyectos de infraestructura en Colombia, especialmente los regulatorios, los comerciales y los de fuerza mayor, son importantes. En el caso de la regulación, esta se ha desarrollado en forma desigual en los diversos sectores. Las instituciones regulatorias son recientes (creadas en 1993 y 1994), sufren periódicamente interferencias políticas, las regulaciones sobre ajuste de tarifas a costos se postergan periódicamente, al igual que aquellas que racionalizan el esquema de subsidios cruzados, optándose por esquemas de ajuste con mayor gradualidad. Adicionalmente, la viabilidad del esquema de subsidios adoptado -Fondos de Solidaridad y Redistribución- es incierta (1999, p. 10).

La consecuencia lógica del proceso referido en cuanto a la edificación, congeló la disposición de créditos para los compradores de vivienda, y en algún momento, los constructores ajenos a dicha involución del mercado terminaron acumulando grandes inventarios de propiedades, que al final tuvieron que ser vendidas por valores por debajo de la ganancia media, por cuanto el sector financiero observaría con recelo el riesgo que implicaba otorgar créditos a una actividad cuya exposición a la volatilidad impediría cualquier inyección capital y, por ende, la disparidad de expectativas terminaría por desanimar a la actividad.

Adicionalmente, en la última década los bancos comerciales cambiaron su principal estrategia de negocio en la industria de servicios financieros, contrayendo el financiamiento de proyectos de infraestructura en países en desarrollo. Esto es relevante, porque en el pasado la banca comercial financiaba proyectos en infraestructura en países en desarrollo principalmente cuando contaban con el respaldo de Organismos de Crédito a la Exportación (OCE) o Agencias Multilaterales. Ante este panorama, se torna imprescindible desarrollar estructuras efectivas de transacción para obtener financiamiento en el mercado de capitales, posiblemente generando nuevas formas de mitigación del riesgo y recobrando el acceso al mercado de capitales en los países de la región. Considerando que los mercados de capitales internacionales proporcionan la mayor fuente de financiamiento y los mercados de capitales locales son una fuente de financiamiento sin explotar para proyectos de infraestructura en la mayoría de los países de la región (Livert, 2007, p.74).

El estrangulamiento del sector constructor edificador para finales del siglo XX en Colombia movilizaría no solo el cambio de legislación, sino la aparición de una serie 
de mecanismos, como los ahorros programados para la financiación de vivienda (AFC), exenciones para aquellos ahorradores cuyos depósitos en calidad de cesantías o ahorros programados estuviesen dirigidos a la adquisición de vivienda. La medida tuvo un impacto trascendental en la oferta de construcción por encima de las viviendas de interés social (VIS); no obstante, estas últimas tuvieron que ser apalancadas de nuevo con subsidios. Reitera Asensio:

Los cronogramas diseñados por los ingenieros y arquitectos sufren modificaciones, los políticos dan explicaciones como pueden, y el ciudadano se impacienta. No hay duda: ejecutar obras requiere una planificación exquisita y un alto grado de compromiso en función de criterios técnicos y, por qué no reconocerlo, políticos (2006, p. 149).

La posición tomada por el Estado en materia de construcción y financiación de vivienda pasaría no solamente por cuenta del apoyo directo del Estado en los últimos años, sino que se convertiría en la línea esencial del programa de gobierno del presidente Santos que tomando, como referencia el caso de países como Brasil entre otros, comenzaría a promover la incubación de programas de construcción de vivienda de interés prioritario (VIP), en las que además de comprometer recursos de la nación, las gobernaciones y las alcaldías tendrían que disponer lotes para el efecto y la identificación de la población habilitada. Según Asensio:

\begin{abstract}
De todas las prioridades políticas que declaran los alcaldes cuando hacen balance de su gestión municipal, el urbanismo y las infraestructuras son temas que siempre están presentes. Una de las claves de la planificación local se encuentra en la ordenación del territorio. El término municipal se encuentra atendiendo a la tradicional clasificación del suelo urbano (consolidado o pendiente por urbanizar) y no urbanizable. También son fundamentales las calificaciones de ese suelo en distintos usos: residencial, industrial, equipamiento deportivo o social, centros educativos, viales, zonas verdes, etc. (2006, pp. 135-136).
\end{abstract}

El campo correspondiente a los proyectos de infraestructura obedece a los temas de equipamiento físico y urbano demandado particularmente por las concentraciones poblaciones, y que bajo condiciones económicas, presupuestales y técnicas terminan siendo ejecutadas por empresas dedicadas no solo a la instalación de los mismos, sino a la operatividad en 
muchos de casos de dichas obras. Esta clase de intervenciones pasa por el tamiz de las convocatorias públicas, sujetos a la disposición de recursos en tanto oferta pública como por el mecanismo de subasta al cual responden particularmente quienes tienen en su haber la experiencia en proyectos de infraestructura física. Argumenta Tamayo y Hernández:

Los estados y municipios sólo tienen acceso limitado al financiamiento de deuda para proyectos de infraestructura, aunque recientemente el mercado ha experimentado una expansión dinámica. Los bancos de desarrollo sólo cuentan con fondos limitados y todavía hay asuntos importantes relacionados con el riesgo que impiden los préstamos de bancos comerciales y la participación de los mercados de capital. En general, los ingresos propios de los municipios son verdaderamente insignificantes (2006, p. 2).

La tradición en este tipo de ámbitos ha estado caracterizada por la disposición de recursos, que a manera de anticipos han sido otorgados por el Estado en el caso colombiano, para que entre tanto, las empresas de infraestructura tuviesen además de un estimulo, un impulso directo a las necesidades iniciales de capital. Sin embargo, pese a la disposición de recursos, el escenario ha indicado que muchas de las empresas dedicadas a este sector no cuentan con la raigambre que se requiere en materia financiera para ser solventes ante cualquier alteración o necesidad que el proyecto propiamente exija o implique. Así, Ejemplifica

A pesar de los múltiples problemas de riesgo genérico para la participación privada en infraestructura en Colombia, la creación de un mercado en generación con participación creciente del capital extranjero, hacen cada vez más remotas las posibilidades de reversión al esquema anterior. La creación del Cargo por Capacidad mejoró las posibilidades de que una planta eficiente se remunere con la difícil señal de precio spot del Pool colombiano (Benavides, 1997). Por ejemplo, una planta de gas a ciclo abierto, que es la de menor costo de capital, puede ingresar al mercado sin contratos y remunerarse. La dinámica del mercado eléctrico colombiano ha generado posibilidades de ingreso que deben tenerse en cuenta antes de formular alternativas genéricas (Alonso et. al:, 2001, p. 104).

La presencia de la banca privada en este tipo de intervenciones ha sido evidentemente poco notable. Las empresas de construcción de obras de infraestructura física, a diferencia 
de las civiles, consagradas comúnmente en el terreno de los equipamientos de vivienda y de las instalaciones conexas, han sido las que, a expensas de la poca capacidad del Estado para acometerlas directamente, han aprovechado para sacar ganancias importantes de las mismas, bajo condiciones no reciprocas con respecto a la magnitud de las obras consagradas en los proyectos y los riesgos inherentes a ellas. Plantean en el Simposio Asobancaria y Banca de Inversión de Bancolombia:

El riesgo construcción es inherente a los diferentes proyectos de infraestructura y se puede dividir en dos grandes categorías: riesgos de sobrecostos y riesgo de mayores plazos, que pueden estar relacionados bien sea con el constructor de la obra, las condiciones del terreno o con eventos externos. Aunque bien los proyectos de infraestructura tiene implícitos ciertos riesgos, los inversionistas institucionales son compensados por las atractivas relaciones riesgo-retorno, con baja volatilidad y baja correlación en comparación con otras clases de activos. Para profundizar la participación de los inversionistas institucionales se requiere de una correcta asignación y mitigación de los riesgos de los proyectos, así como una correcta estructura financiera que genere confianza a los acreedores (2011, p. 10).

El patrón característico de la infraestructura física es que la valoración que se hace de las acometidas e intervenciones directas en la mayoría de los casos no atina a las proyecciones estimadas. De allí que, para cualquier prestamista de primer o segundo piso, cualquier incursión en este tipo de inversiones podría acarrearle problemas financieros y de liquidez. La banca en general, a excepción de la pública, por lo menos en Colombia, se ha hecho al margen de patrocinar en gran medida obras cuyas valoraciones son poco exactas, y que al margen de sus fines, podrían generar dificultades directas al sistema financiero (figura 2). 


\section{Retornos en infraestructura por sectores}

Sectores Globales de Infraestructura

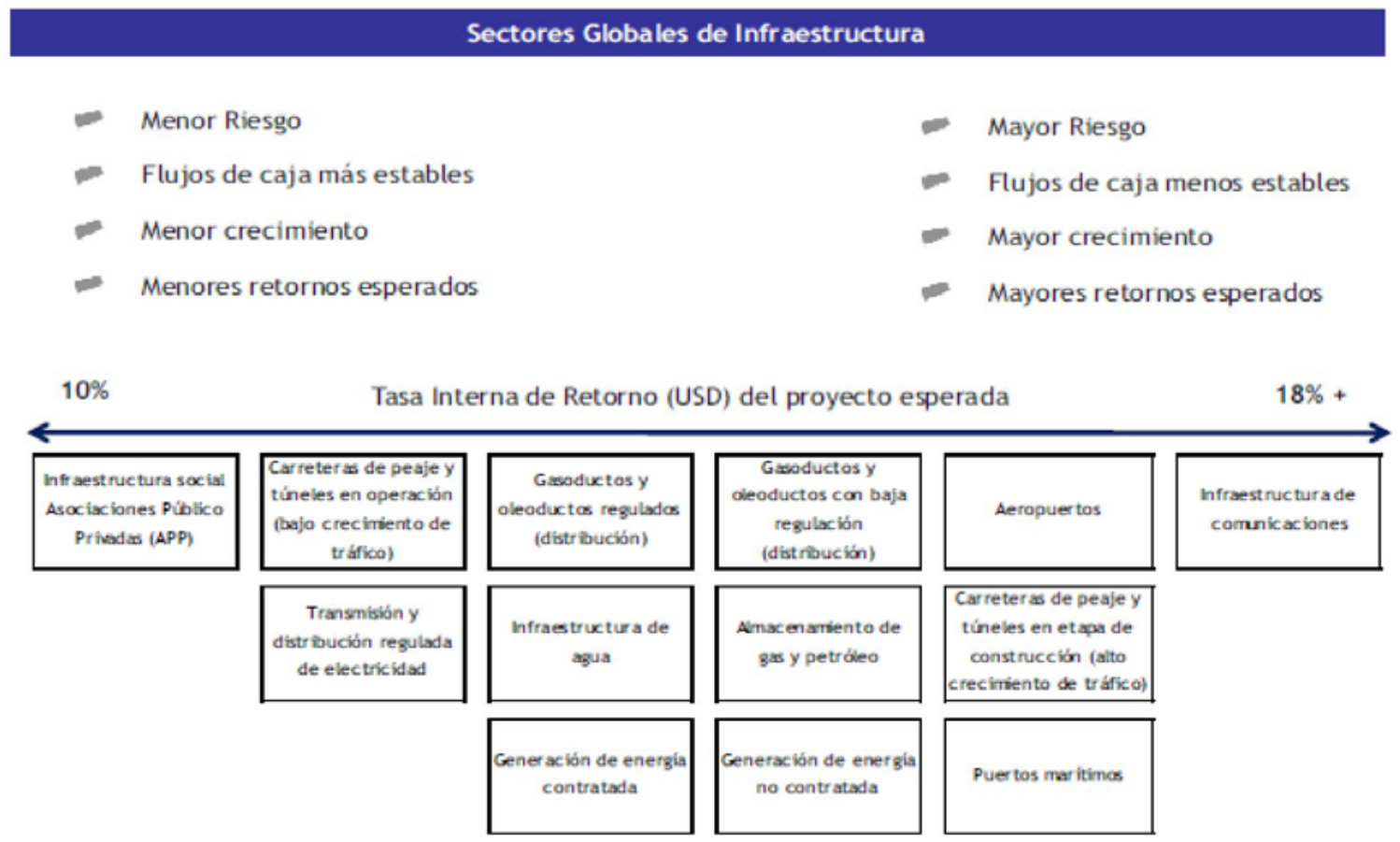

Figura 2. Retornos en infraestructura por sectores

Fuente: Simposio Asobancaria y Banca de Inversión de Bancolombia (2011).

Es la aversión al riesgo la que constituye el mecanismo no propiamente financiero que complementa la disposición de recursos para la financiación de proyectos civiles y de infraestructura. En este sentido, las tasas de los préstamos otorgados para el desarrollo de los mismos en la mayoría de los casos no solo contemplan la tasa de ganancia esperada por las instituciones financieras, sino que se agregan valoraciones sujetas a los posibles altibajos que puedan ocurrir (especulaciones) y al establecimiento del valor dinero en el tiempo (acumulación), con lo cual la posibilidad de acceder a los créditos está más del lado de las expectativas del retorno financiero del proyecto (figura 3). 


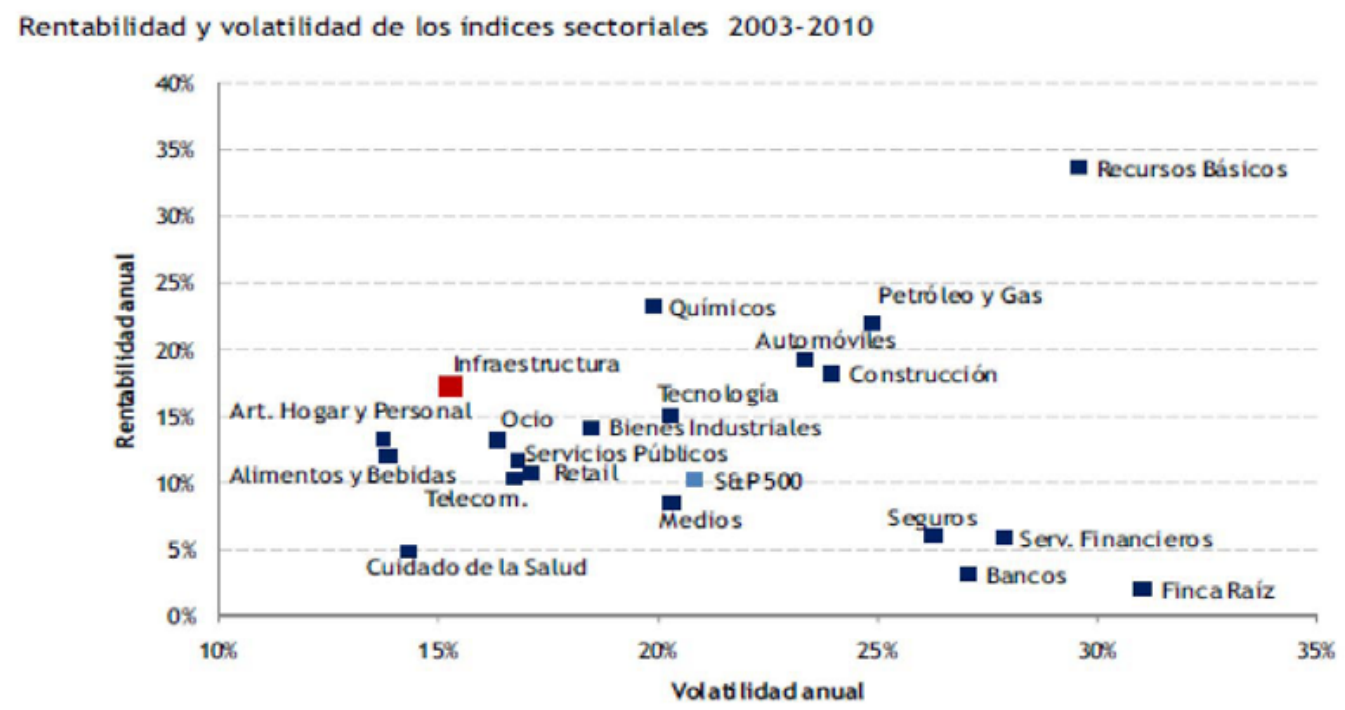

Basado en el comportamiento de indicadores accionarios sectoriales (Dow Jones Titans Indexes), se puede af irmar que la infraestructura presenta una relación riesgo retomo más eficiente que la de otros sectores de la economia. La participación en este sector puede aportar a la optimización de las características de los portafolios de inversión.

Gráfico 3. Relación riesgo-entorno

Fuente: Simposio Asobancaria y Banca de Inversión de Bancolombia (2011).

La exposición a la volatilidad financiera arrojada por las diferentes interacciones en que se desarrolla la actividad de infraestructura, corresponde al cúmulo de factores y gradientes sobre los que es evaluada la posibilidad de que cualquiera de los proyectos incubados en ellos tenga un alcance exitoso. Es decir, que la disposición al crédito no pasa por la oferta de liquidez o animadversión a los proyectos básicos, civiles o de infraestructura a la par con los grandes megaproyectos. La intención de abarcar crediticia y financieramente depende de la volatilidad y la cobertura del riesgo, que de no lograrse, restringe a la banca en general para poder orientar recursos hacia ellos. 


\section{Acceso, Intervención y Mecanismos de Financiación de la Banca Multilateral en Proyectos de Infraestructura}

La Banca Multilateral emerge como estructura cuya base de recursos proviene de los ahorros, aportes, fondos y líneas de financiación creadas institucionalmente por los organismos y países pertenecientes a ella. Normalmente responden a desembolsos de recursos otorgados en calidad de deuda externa, prestamos interestatales o formulas financieras planteadas por los mismos organismos, para garantizar no solo la ejecución efectiva de los recursos, sino la recuperación en el tiempo de los recursos desembolsados, razón económico-financiera que no es contemplada por la banca privada. Así, explica Livert

Los bancos multilaterales de desarrollo (BMD) son intermediarios financieros internacionales cuyos accionistas incluyen a los "prestatarios" (países en desarrollo) y los "contribuyentes" o "donantes" (países desarrollados). Estos han tenido un impacto significativo en el desempeño económico y social de los países de América Latina y el Caribe, mediante el financiamiento directo de proyectos y programas o a través de movilización de otras fuentes de financiamiento público y privado. Además BMD pone a disposición servicios complementarios como asistencia técnica, información estadística, diálogos sobre políticas económicas, manejo de fondos fiduciarios, estudios sobre desarrollo, evaluación del gasto público, capacitación de funcionarios, donaciones para financiar bienes públicos internacionales, etc. Los BMD proveen préstamos de largo plazo a tasas de interés generalmente más favorables que las del mercado para la gran mayoría de países prestatarios, préstamos concesionales a tasas de interés muy bajas y períodos largos de repago, provisión de garantías para la inversión privada, además de donaciones dedicadas principalmente a la asistencia técnica, entrenamiento y creación de capacidad en los países prestatarios. LOS BMD cuentan con la característica de ser acreedores preferenciales en su relación con los prestatarios, estando en una situación más favorable que los acreedores privados $(2011$, p. 30).

En términos generales, el grueso de intervenciones en diferentes megaproyectos llevados a cabo en diferentes países por la Banca Multilateral resguardan las condiciones financieras sobre las que se desarrollan los mismos. En este sentido, más que la cobertura de 
los riesgos o la disposición de grandes cuantías, la participación directa de la Banca tiene como premisa la garantía, fluidez y respaldo en cuanto a los mecanismos de financiación que esta pueda estimar para realizar los mismos. Es decir que no solo funciona como prestamista, sino que hace las veces de banca de capital y de inversión.

Por ejemplo, y a modo de ilustración, la ventaja del Banco Mundial reside en que puede financiar proyectos de gran magnitud, movilizar una considerable cantidad de recursos de desembolso rápido en situaciones de crisis, y ofrecer conocimientos sobre la base de experiencias en otras regiones del mundo. Por su parte el Banco Interamericano de Desarrollo ha estado, a lo largo de toda su existencia, en estrecho contacto con los países de la región y ha acumulado un profundo conocimiento acerca de su realidad social, institucional y política, lo que le confiere una ventaja en las operaciones destinadas a los sectores sociales, los programas de reformas institucionales (reforma del Estado y de la administración pública) y en temas de gobernabilidad. La literatura especializada en financiamiento para el desarrollo, ha evidenciado en reiteradas oportunidades, el rol del sistema financiero como mecanismo para encauzar de manera eficiente el ahorro tanto para financiar la inversión y la innovación tecnológica, como para facilitar el acceso al financiamiento de los diversos agentes productivos. Específicamente, el desarrollo del sector financiero contribuye al crecimiento económico porque reduce el costo del capital y mejora la asignación de recursos (aumentando la cantidad y calidad de los proyectos de inversión) (Livert, 2011, 33-34).

La garantía de los recursos girados por dichos entes corresponde al paquete de endeudamiento, empréstitos o servicio a la deuda sobre la cual debe responder el país que solicita dicha intervención con mecanismos que superan las bondades y condiciones de la banca tradicional. En comparación, cuando se trata de evidenciar la capacidad de financiación y financiera de la banca comercial o similar, es más que conocida la poca capacidad que tiene esta para encarar proyectos que exijan más que la sola disposición de recursos. En términos generales, la banca privada no está para compulsar megaproyectos; según el BID:

La disponibilidad de financiación de largo plazo es esencial para los proyectos de infraestructuras, caracterizados por grandes inversiones de largo periodo de maduración. Por desgracia, los mercados locales de capitales de los países IIRSA rara vez ofrecen tales plazos de financiación, lo que entrañara un grave riesgo de refinanciación 
si el proyecto se financia en dichos mercados. Ello exigirá, en todo caso, contar en todo caso con garantías adicionales de los países involucrados en cada proyecto, de agencias multilaterales, o instituciones financieras (2002, p. 12).

El alcance que tiene la Banca Multilateral frente a la tradicional sobrepasa el pulso de las expectativas. Mientras que la primera dimensiona la correspondencia, impacto, medición y evaluación en distintos frentes, no solo el económico y el financiero, la Banca privada estima la correspondencia entre los requerimientos de la inversión y la capitalización del recurso puesto en la misma, es decir que, el papel de esta última radica en focalizar recursos en fuentes que garanticen además de la redención del capital, el retorno de ganancias, debido precisamente a que el fin de esta es colocar dinero no prestarlo, ni mucho menos involucrarse en iniciativas ajenas a las finanzas bancarias. De esta manera, como lo exponen Rojas y Rodríguez:

Como respuesta a las necesidades que afrontan las economías nacionales en el nuevo contexto de la globalización, y en particular la necesidad de contar con infraestructuras que faciliten el libre comercio, los Estados y los particulares han desarrollado estructuras financieras y jurídicas que hacen posible que tanto los Estados como los mismos particulares construyan o instalen estructuras (líneas férreas, túneles, aeropuertos, autopistas, acueductos), sin desembolsar grandes cantidades de recursos del erario público6. Esas nuevas estructuras se agrupan en lo que hoy conocemos como "Project Finance", concepto éste que significa un innovador mecanismo de financiación que permite al empleador del proyecto, público o privado, llevarlo a cabo obteniendo la financiación de la inversión sin contar, por otros medios propios o ajenos, con la capacidad financiera necesaria. A diferencia de los mecanismos de crédito utilizados habitualmente, el Project Finance se basa fundamentalmente en la capacidad que tiene el proyecto para generar recursos, que habrán de ser suficientes para pagar los rendimientos del capital, el beneficio del explotador y la devolución del capital invertido (2008, p. 1-2).

El equipamiento, las acometidas, los derrames e impactos de los proyectos de infraestructura catalizan las condiciones financieras que sirven para valorar las condiciones sobre las que se detallan dichos proyectos. A pesar de ello, las especificaciones técnicas y las estructuras sobre las cuales son puestos en marcha los proyectos, constituyen elementos que aún no 
pasan por las evaluaciones financieras de las entidades que con cargo a líneas de crédito en la materia avisten la posibilidad de orientar recursos hacia dichos menesteres. No obstante, la revisión del tema entre las partes es de carácter discrecional. Argumenta Naranjo:

La construcción es una actividad que demanda altos niveles de apalancamiento. Son varios los mecanismos a través de los cuales el sector obtiene financiamiento de acuerdo a la obra a realizarse: financiamiento de organismos internacionales para obras de infraestructura pública; crédito bancario para vivienda o edificaciones; operaciones de fideicomiso; y, crédito directo otorgado por los constructores (2011, p. 7).

Los megaproyectos obedecen mas a planes estratégicos de fondo, cuya naturaleza puede ser mediada por diversos intereses como lo son los binacionales y organizaciones multilaterales. Se debe anexar, además, otro factor existente como consecuencia de los acuerdos de libre comercio, los cuales exigen plenamente ingentes esfuerzos para la instalación de grandes obras que permitan la movilidad de bienes, personas y recursos. Estos finalmente terminan siendo contestados por la Banca Multilateral, que normalmente tiene en esencia la capitalización de recursos proveniente de los países y estados miembros de la misma, que en acuerdo hacen efectivo los recursos para ello. Ante esto, analiza Delgado

\footnotetext{
El debate acerca de las fuentes óptimas del financiamiento vial se encuentra en pleno desarrollo a nivel internacional. En este trabajo se presentan las principales líneas argumentales en favor de los esquemas de tributación general, de los impuestos específicos y de los sistemas de peaje, tomando en cuenta tres aspectos básicos: la operatividad de cada fuente de financiamiento, la relación eficiencia-equidad y la existencia de restricciones fiscales, punto esencial en el análisis del caso argentino (1998, p. 11).
}

En este tipo de intervenciones, la financiación termina estando por fuera del alcance de la banca tradicional, crediticia y financiera, que no alcanza ni individualmente ni en bloque a contrarrestar las necesidades que los Estados o que la propia magnitud de la obra indica. Por ende, es de anotar que a mayor tamaño de las megaobras, la distancia entre las fuentes de recursos se vuelve exigua y poco trascendental, de allí que sobresalga la capacidad financiera de la banca conformada por diferentes organismos internacionales, los cuales, a expensas de las necesidades, tratan de fortalecer la institucionalidad con los proyectos. Así, 
Proyectos, muchos de ellos están enfocados en mejorar la calidad actual de las vías y redes. En este caso, siempre y cuando las consecuencias ambientales y sociales, y las condiciones de financiación no sean adversas sería adecuado emprender dichas obras. Sin embargo, existen obras nuevas que implican la intervención directa en áreas específicas (Pacífico, Amazonas), donde se deberían realizar evaluaciones Multicriterio que integren de manera real a las poblaciones y ecosistemas afectados (Sánchez, 2018, p. 19).

El marco de referencias financieras que permiten analizar este tipo de proyectos está relacionado con la magnitud del riesgo a asumir. La banca, tal cual y como se conoce es la más vulnerable al riesgo, no solo por la propia naturaleza de los recursos que utiliza, además de la ampliamente desconocida consideración de los recursos de financiación apreciados en diferentes frentes. La cadena del valor del sector financiero poco se conoce o es distinguida, debido precisamente a la poca correspondencia que se crea entre la liquidez del sector y el margen de retorno que esperan de los demás sectores. Arguye Lucca indica que

En términos generales e independientemente del mecanismo de generación utilizado, los recursos financieros con los que cuenta el sector publico (cualquiera sea su jurisdicción) provienen en última instancia del sector privado. Seis son las formas básicas a través de las cuales los gobiernos locales han obtenido los recursos que les permiten financiar sus actividades:

- Por medio del cobro de tarifas de consumo, también denominadas tasas de uso, que son aplicadas básicamente en aquellos servicios en los cuales el consumo individual es susceptible de ser medido (tales como la electricidad, el agua, las cloacas, las telecomunicaciones y el transporte público).

- Mediante el cobro de impuestos locales, los que se pagan sin tener en cuenta el nivel de uso de los servicios públicos, y entre los que destacan los impuestos personales, a las actividades comerciales y los impuestos prediales (que al decir de especialistas como Kenneth Davey (1998) tienen un significativo potencial para transformarse en un importante medio de financiamiento de las infraestructuras locales).

- A través de financiamiento directo, lo que significa transferir la provisión de servicios públicos e infraestructura al sector privado mediante mecanismos de concesión, inversión conjunta (público privada) y esquemas de construcción, operación y transferencia (COT). 
- Por medio de transferencias intergubernamentales desde los niveles superiores de gobierno hacia el gobierno local, en los cuales dichas jurisdicciones coparticipan la recaudación impositiva con el nivel municipal.

- Mediante el acceso al crédito bancario sea este de carácter local o de organismos multilaterales de crédito (como el caso del BID y del Banco Mundial entre otros), los que son cancelados con la recaudación de tarifas de consumo o de impuestos locales.

- Mediante el acceso directo al mercado de capitales (nacional o internacional), a través de la emisión de bonos y títulos municipales, en los que juega un papel central, tanto para la compra de los bonos como para la fijación de la tasa de interés de los mismos, el análisis crediticio de la viabilidad del municipio (1999, p. 3).

Las grandes obras no hacen parte del interés de los grupos financieros, debido al prolongado tiempo en que estas comienzan a generar frutos, impactos y resultados, oor ende, es de refrendar la idea de que el papel de la banca, por lo menos privada, no es el de capitalizar ningún tipo de obra, megaobras o proyecto, la realidad es que bajo expectativas y proyecciones crean su propio margen de ganancias y de retorno de capital, que no alcanza a ser internalizado por los requirentes de recursos, cuya redención del capital está más expuesto a las atracciones de otros proyectos, que a la meta de cumplir con los presupuestos estimados en primera instancia o los impactos en segunda. Según el consorcio Hugo Millán:

\begin{abstract}
Porción importante de la financiación de proyectos públicos de infraestructura en Colombia se ha concentrado en tres instituciones multilaterales, a las cuales se ha recurrido por las siguientes razones: El gobierno fue el único responsable por el desarrollo y ejecución de este tipo de proyectos por varias décadas, toda vez que su rentabilidad tenía más consideraciones de beneficio social. El sector privado no participó en ellas sino a partir de mediados de la década de los noventas. El gobierno no contaba con recursos propios para adelantar las inversiones. Los préstamos de estas entidades son a largo plazo, es decir entre 10y 20 años de plazo, lo que permite la recuperación de las inversiones (2004, p. 28).
\end{abstract}

La Banca Multilateral percibe recursos de diversas fuentes que depositan en ella los excedentes, inversiones o recursos emanados de actividades cuyo rendimiento en el tiempo también está expuesto a vigencias importantes y que pueden tardar varios años antes de 
su culminación o desarrollo. Particularmente, constituyen un importante amparo para inversiones que requieren relevantes contingentes de recursos, así como respaldos económicos que no se adquieren fácilmente bajo la banca crediticia y financiera, razón por la cual termina siendo considerada una banca de apoyo.

\section{Capacidad y condiciones del sistema financiero colombiano para la realización de megaproyectos y proyectos de construcción e infraestructura}

El sistema financiero colombiano ha estado en gran parte guiado y habilitado por la presencia de la banca pública que, a pesar de los procesos de privatización, aún conserva la disposición de recursos a sectores cuya oferta no alcanza a obtenerse por parte de las entidades privadas. La infraestructura en todas sus manifestaciones siempre ha contado con el concurso de los recursos públicos, a través de marcos jurídicos contractuales tras los que han podido servirse de ellos las componendas financieras, que por medio de bancos de primer y segundo piso las han hecho efectivas. Caracteriza el BID:

\section{El mercado colombiano es muy profundo en deuda pública, con plazos de hasta 10 años, pero muy poco en deuda corporativa. Todas las emisiones de renta fija privada están indiciadas a la inflación, con plazos medio y máximo de 6,5 a 10 años respectivamente (2002, p. 26).}

El limitado margen de maniobra que tienen las empresas dedicadas a la construcción, la infraestructura y los megaproyectos es igualmente comparable con las condiciones financieras de estas, que no contienen un grueso importante de recursos para poder solventarse por cuenta propia, es decir, que aún con o sin la ayuda del Estado o la propia incursión de la banca privada, la composición y estructura financiera de las empresas dedicadas a las intervenciones físicas no se constituye en el patrón característico a resaltar, o por lo menos representa su mayor debilidad a la hora de acceder a créditos. Así: 
En un contexto en el que uno de los principales limitantes del sector inmobiliario en Colombia ha sido la falta de acceso a capital -una característica que ha llevado a que los proyectos tengan que fraccionarse- se hace aún más evidente la necesidad de estar actualizados sobre el desarrollo de las nuevas alternativas de inversión. Estas últimas sirven como un puente entre el sector inmobiliario y el mercado de capitales, y su materialización plantea una serie de retos claramente identificables (Cárdenas y Castellanos, 2008, p. 1).

La consideración de la estructura financiera de las compañías dedicadas a estas actividades debe ser el punto central de la discusión. En este sentido, es de reconocer que el tamaño de las obras en un escenario de baja reciprocidad en cuanto a los activos y patrimonio de las empresas de dichos sectores, ya que representa para las propias entidades financieras el más claro manifiesto de las restricciones a las que están expuestas, y de allí su dilación para no ofertar recursos a dichos frentes. Como propone Blázquez,

En relación con esta falta de volumen se encuentra otra de las debilidades a las que se enfrenta la empresa de ingeniería tradicional, que es la dificultad para poder ofrecer financiación. En un contexto económico en el que el gobierno está apostando cada vez más por las fórmulas de financiación público-privado, ser una empresa grande, con capacidad de financiación y asunción de riesgos es muy determinante para ganar cuota de mercado. Esto ha dado como resultado que la empresa canadiense haya participado más como compañía subcontratada que como contratista general, tendencia que por otro lado está cambiando progresivamente (2012, p. 60).

Si bien el Estado colombiano ha tratado de mejorar en los temas que conciernen a la estructuración de las compañías y del marco legal que rige los esquemas de contratación de las empresas dedicadas al desarrollo de obras, proyectos o megaobras, la respuesta para el efecto ha sido lenta debido precisamente a que el reconocimiento de grandes jugadores en materia de empresas de construcción o infraestructura apenas aparece en el foco de interés de las entidades financieras, a la par con las expectativas del Estado respecto a ellas, con las que se pretende que sean más socias de los proyectos que oferentes. Estima Serrano:

Constructores de proyectos de obras públicas. Con recursos propios o con recursos de crédito, en especial si el constructor hace parte de un grupo económico 
con músculo financiero para conseguir los recursos que demanda el proyecto. Sin duda, se han fortalecido patrimonialmente y algunos comienzan a reorientarse hacia inversionistas de largo plazo bajo esquemas de financiamiento de proyectos (Project Finance) (2010, p. 110).

En comparación, la clase de mercados que manejan unos y otros dista en el margen de expectativas que se crea en un proyecto de magnitudes relevantes y las consideraciones que con respecto a ello esperan las entidades financieras y los empresarios de obras. Los primeros estiman conveniente tamizar la disposición de recursos conforme al reintegro en un mediano plazo de los recursos desembolsados y los segundos aspiran a que las obras tengan un mayor aliento para obtener mayores recursos en el tiempo; frente a ello, mientras que la recuperación del capital en préstamo se convierte en el centro de las entidades financieras, para los empresarios se explica por el tiempo. Contextualiza el Consorcio Millán:

La naturaleza de largo plazo de los proyectos de infraestructura exige que su financiación tenga también plazos amplios para evitar refinanciaciones costosas. En vista de que en Colombia no está plenamente desarrollado un mercado de capitales que permita financiar proyectos de infraestructura con plazos adecuados, se recomienda recurrir a esquemas de emisión de bonos como los sugeridos con técnicas unbundling. Estos bonos normalmente requerirán una alta calidad financiera que se logra con adecuadas garantías de agencias multilaterales o de instituciones financieras de primer orden (2004, p. 12).

La definición de tasas de ganancias por parte de las entidades financieras por encima de las expectativas de tiempo que conforme a desembolsos esperan recibir los empresarios de la construcción, despliegan las condiciones de los indicadores, parámetros y variables que distancian la posibilidad de que ambos sectores puedan encontrarse. Mientras que la actividad edificadora realiza actividades para obtener réditos consecuentes a la actividad de la construcción o infraestructura cuyo monto justifica la espera, la banca desembolsa recursos en la medida en que las tasas de ganancias superan los montos desembolsados. Diserta FIAP: 
COLOMBIA - Los beneficios asociados a la inversión en infraestructura para los fondos de pensiones son el acceso a inversiones atractivas de largo plazo acordes con el objetivo de pensión; flujos estables cubiertos contra la inflación; y ganancias generadas por la diversificación derivada de la baja correlación de las inversiones en infraestructura con los activos financieros. Por su parte, los beneficios generados por los fondos inmobiliarios están dados por un mayor grado de diversificación, gracias a la menor cuantía de los proyectos y mayor oferta de los mismos; la concentración de las inversiones en activos asociados al desarrollo de actividades comerciales, lo cual facilita su valoración y su liquidación; así como las posibilidades de rendimientos adicionales a partir de la valorización de los activos (2011, p. 49).

En cuanto a la comparación que uno y otro sector hacen a la hora de estimar la conveniencia de acceder o negar la posibilidad de recursos, se incuba la necesidad de analizar el alcance que tiene la discrecionalidad con la que ambos miden los parámetros sobre los cuales actúan. Por eso es importante aclarar que, ante la falta de recursos suficientes por parte de las empresas constructoras y edificadoras colombianas tendrán que replantear su quehacer, expuesto a exigencias que superan las solas especificaciones complementarias y técnicas de la obras, las cuales, para ser solventadas, exigirán importantes esfuerzos en materia de recursos, como los vinculados a los fondos de cesantías, pensiones y ahorros.

Los fondos de pensiones en este país vienen contribuyendo activamente en el desarrollo económico, canalizando de manera directa recursos a sectores productivos claves. Dentro de ello, ya se ha venido financiando el desarrollo de proyectos de infraestructura, aunque de manera indirecta, es decir, a través de la inversión en bonos y acciones de empresas, cuyo principal destino estaba destinado a ese objetivo. Se estima que actualmente la inversión de las AFP en infraestructura, de manera indirecta, se ubica en US\$9.591 millones, representando esta el 18,7\% del valor de los fondos de pensiones, equivalente al 3,5\% del PIB. Es de anotar que el sector con mayor participación ha sido el eléctrico energético, representando cerca del $84 \%$ de la inversión de los fondos de pensiones en infraestructuras. Es así como los fondos de pensiones tienen una participación accionaria importante en empresas del sector como ISA, Ecopetrol e Isagen con unos porcentajes del $17,5 \%, 4,2 \%$ y $11,6 \%$, respectivamente (Dos Santos et al., 2011, p. 13). 
Es importante que sean definidos los aspectos con los cuales puedan ser mejoradas las condiciones crediticias, financieras y de recursos de las empresas dedicadas a la actividad constructora y edificadora. Ello se logra, en la medida que se amplíe la presencia de empresas extranjeras en alianzas con las locales o que se inculque la necesidad de consolidar patrimonialmente los recursos de las mismas, a través de la inversión en bolsa de valores o conforme al concurso de estas actividades cuyos réditos igualmente están sujetos a la dependencia del tiempo y de los desembolsos, más que a las tasas de ganancias y redenciones mediatas, con el objetivo de que estas puedan solventar los requerimientos de los proyectos. Propone Naranjo:

Uno de los factores favorables para el crecimiento del sector ha sido el otorgamiento de un mayor monto de crédito, especialmente por parte de los bancos privados. Cada vez ha sido mayor la participación del crédito otorgado para vivienda dentro de la cartera total del sistema bancario. La estabilidad monetaria ha permitido que el sistema bancario considere atractivo el financiamiento de esta actividad. Otro mecanismo de financiamiento que ha tenido gran auge en los últimos años, especialmente para llevar a cabo proyectos de construcción de edificaciones, es la constitución de fideicomisos. Por lo general, los dueños de los terrenos ceden en fideicomiso su propiedad, lo que garantiza que no se perderá el capital de riesgo inicial. Por último, existe una notable tendencia a que los promotores de proyectos financien la venta directa de los mismos. Este mecanismo brinda al comprador una mayor facilidad de acceso; sin embargo, el plazo del crédito es menor en comparación a los otorgados por instituciones financieras (El Comercio, citado por Naranjo, 2011, p. 7).

La contratación de créditos, financiación y complementación de recursos con la banca multilateral también hace parte de la bandeja de posibilidades que podrían atender las necesidades de recursos de las compañías. No obstante, el lastre de estas radica en que no cuentan con la suficiente capacidad, experiencia o vigencia para alcanzar dichas entidades con solidez, precisamente por la exposición al riesgo, la inestabilidad patrimonial a la que están expuestas y la evidente iliquidez que, al parecer, constituye un patrón característico de estas empresas, las cuales, a diferencia de las financieras, canalizan diversas fuentes de recursos, no necesariamente en efectivo, para poder cumplir con sus obligaciones o el desarrollo de las obras. Arguye el Consorcio Hugo Millán: 
La financiación de proyectos de infraestructura con divisas extranjeras trae consigo la necesidad de diseñar esquemas para cubrirse del riesgo cambiario, toda vez que los ingresos de los proyectos serán en moneda local. El riesgo cambiario será significativamente importante cuando se presenta una disparidad severa entre el flujo de los ingresos del proyecto que normalmente estará en moneda local y el de la deuda externa que estará en divisas extranjeras (2004, p. 12).

La banca crediticia y financiera debe apostar a la inclusión de sectores dinámicos como el constructor, edificador y de grandes obras, precisamente por la curva de beneficios que podría traer no solo en materia de clientes y flujos de dinero, sino por la necesidad que tienen las mismas entidades de consolidarse no solo en calidad de captadoras y colocadoras de recursos sino también como instituciones eficientes, productivas y con resultados a la hora de apostarle bajo alguna asignación o participación a proyectos que requieren apuestas financieras directas o del acompañamiento en cuanto a asesorías financieras. Según la ONU:

En la mayoría de las economías en desarrollo, los servicios financieros sólo están disponibles para una minoría de la población, con frecuencia para una pequeñísima minoría. Aunque los sectores financieros se están ampliando a medida que estas economías crecen, los activos usualmente permanecen concentrados en las manos de unos pocos. La mayoría de las personas en los países en desarrollo no tienen cuentas de ahorros, no reciben crédito de una institución financiera formal y tampoco tienen pólizas de seguros. Raramente realizan o reciben pagos a través de las instituciones financieras. De hecho, la mayoría, en casi todos los países, raramente ingresa a sus predios (2006, p. 1).

El desempeño crediticio, financiero y de financiación del sistema colombiano ha estado guiado por las tasas de ganancias por encima del rendimiento del capital. En términos generales, para la banca privada resulta más atractivo el ponderar la tasa de ganancia por encima de la redención y rentabilidad del dinero invertido; este comportamiento termina ubicando al sistema financiero en un subastador de la probabilidad de éxito de un sector económico, a medida que extiende recursos conforme al margen de la tasa esperada o que se surge precisamente por parámetros y evaluaciones. 
La disposición de la banca y del sistema financiero colombiano está guiada por los procesos de acumulación, animadversión al riesgo y pronta recuperación de los capitales invertidos, independiente de la actividad. Esto pone en evidencia que el interés de la banca consiste en disponer recursos, solo en la medida en que la movilidad de estos demuestre un retorno rápido de la inversión ofrecida en crédito.

El escenario de análisis está explicito a la hora de congraciar las oportunidades que tiene el sector constructor, edificador y de proyectos con las estructuras crediticias, financieras y de recursos provistas por las entidades y organismos multilaterales en la materia. No obstante, es importante identificar las circunstancias y momentos en los que las expectativas y estipulaciones de los mismos rompen cualquier posibilidad de hallar equilibrio entre los recursos disponibles y las necesidades que puedan tener los requirentes de grandes obras. El tema pasa por la revisión de las condiciones de ofertas, subastas y capacidad de asociación entre ellos conforme a los proyectos.

\section{Conclusiones}

El estudio sobre el mercado de activos, desembolsos y recursos de inversión dirigido a proyectos de infraestructura constituye una base importante de reflexión, más aun cuando en el país y varias naciones de Suramérica vienen postulándose diversos programas y proyectos de obras de gran magnitud, en las que, si bien prevalece la coordinación con organismos multilaterales, la presencia y participación de la inversión privada en ello requiere una revisión y postulación de propuestas en materia de obtención, gestión y manejo de recursos crediticios y financieros, que también en la mayoría de los casos estiman la conveniencia de analizar dicho terreno.

La revisión de los proyectos de infraestructura en materia de financiación no constituye solo la interpretación de los componentes financieros que obedece a la edificación como tal. En el momento, y por cuenta de las necesidades financieras de los distintos entes territoriales, es reconocer los beneficios económicos y de rentabilidad que trae para los entes territoriales locales y regionales contar con infraestructuras que rebasan en beneficios, exigencias e impactos su sola disposición; en la capacidad de generar recursos territoriales 
vía obras de infraestructura estará también afincada la posibilidad de que la banca dirija su mirada a dicho campo.

La evaluación de proyectos de infraestructura debe regresar de nuevo a la revisión académica e investigativa de las aulas. No se trata solo de determinar la relación costo-beneficio, sino también de estimar de manera integral las exigencias que desde diversos rubros convoca la instalación o realización de obras de carácter ingenieril, y que aparentemente terminan cuando estas son concluidas o cuando los presupuestos se acaban. En la literatura general de proyectos, debe afianzarse aún más la revisión de proyectos de carácter estratégico o interestatal, como los que aparecen con cada puesta de integración y que escapan a la revisión profesional en las aulas.

\section{Referencias}

Alonso J. C., Benavides J., Fainboim Yaker I., \& Rodríguez C. J. (2001). Banco Interamericano de Desarrollo. Participación privada en proyectos de infraestructura y determinantes de los esquemas contractuales adoptados: el caso colombiano. Washington D.C.: Fedesarrollo.

Amador Cabra L. E. (2011). Los servicios públicos domiciliarios frente a las reformas económicas en Colombia (Primera Edición). Bogotá: Universidad Externado de Colombia.

Asensio Romero, P. (2006). El libro de la gestión municipal. Claves de éxito para políticos y directivos locales. Madrid: Ediciones Díaz de Santos.

Banco Interamericano de Desarrollo. (2000). Un nuevo impulso para la integración de la infraestructura regional en América del Sur. Recuperado de http//www.iadb.org/intal/ publicaciones/infraestructura/

Banco Interamericano de Desarrollo. (2002). Financiación de proyectos transnacionales de Infraestructuras en América del Sur (Iniciativa IIRSA). Grupo Analistas Financieros Internacionales.

Banco Mundial. (1994). Informe sobre el desarrollo mundial, infraestructura y desarrollo. Washington.

Benavides, J. (1997). "Capacity Payments in Predominantly Hydroelectric Electricity Markets: The Case of Colombia”. En $19^{a}$ reunión anual de la USAEE/IAEE (International Association of Energy Economics), San Francisco, Estados Unidos. 
Blázquez J. (2012). Sector de la arquitectura, la ingeniería y la construcción en Canadá. Oficina Económica y Comercial de la Embajada de España en Ottawa. ICEX. 1-168.

Carciofi A. (2006). Iniciativa Para la Integración de la Infraestructura regional de América del Sur (IIRSA). Aspectos conceptuales e Institucionales. Cátedra Andrés Bello. Buenos Aires: Universidad Nacional de Rosario.

Cárdenas A. I., \& Castellanos P. (2008). Alternativas para financiar la construcción. Camacol, informe económico (2008). En 4 Seminario Alternativas del mercado de valores para financiar la construcción, organizado por CAMACOL, la Bolsa de Valores de Colombia y el Consejo Privado de Competitividad, Bogotá, Colombia.

Consorcio Hugo Millán (2004). Plan de Infraestructura de Transporte y Portuaria Para el Desarrollo Minero en Colombia. Informe Final Modulo II. Unidad de Planeación Minero Energética -UPME- 1-80.

Delgado R. (1998). Inversiones en infraestructura vial: la experiencia Argentina. Serie Reformas Económicas 6, 1-46.

Dos Santos E., Torres D., \& Tuesta D. (2011). Una revisión de los avances en la inversión en infraestructura en Latinoamérica y el papel de los fondos de pensiones privados. BBVA. Research. Análisis Económico 11/36 Documentos de Trabajo.

Fainboim Yaker, I., \& Rodríguez Restrepo, C. (1999). El desarrollo de la infraestructura en Colombia en la Década de los noventa Parte I. Serie Reformas Económicas 51. CEPAL.

FIAP (2011). Fondos de Pensiones: Inversión en Vivienda e Infraestructura. Serie Regulaciones Comparadas, 1-57.

Livert-Aquino, F. (2011). Ecoeficiencia y desarrollo de infraestructura urbana sostenible en Asia y América Latina. Estudio de mecanismos de financiamiento de la infraestructura urbana utilizando criterios de ecoeficiencia. Naciones Unidas, Comisión Económica para América Latina y el Caribe (CEPAL), 1-87.

Livert Aquino, F. (2007). Mecanismos de financiamiento de la infraestructura urbana utilizando criterios de eco-eficiencia. Documento de proyecto. Comisión Económica para América Latina y el Caribe (CEPAL). Informe final. Santiago de Chile: Naciones Unidas.

Llanes, M. C. (2011) Desarrollo de los fondos de pensiones y la inversión en proyectos de infraestructura. Borrador. Mayo.

Lucca, C. (1999). Mecanismos no tradicionales de financiamiento de obras de infraestructura y equipamiento en municipios. En XIV Seminario Regional de Política Fiscal, Santiago de Chile, Chile. 
Motenzuma, E. R. (2008). Infraestructura. Proyecto (2008). Piensa Colombia: los aportes de la academia (Tomo 1. Vol. 7). Documentos de política pública. Senado de la República y Universidad Nacional de Colombia.

Naranjo, M. (2011). Boletín de Análisis Sectorial y de MIPYMES. Sector de la Construcción. Centro de Investigaciones Económicas y de la Micro, Pequeña y Mediana Empresas. Programa de Economía. FLACSO Sede Ecuador. Ministerio de Industria y Productividad. $1-23$.

ONU. (2006). La construcción de sectores financieros incluyentes para el desarrollo. Nueva York: Naciones Unidas.

Rojas Tamayo, A., \& Rodríguez Fernández, M. (2008). Tres Sistemas para la Determinación del precio y Condiciones de Pago en el contrato internacional de construcción.REVIST@ e-Mercatoria, 7(1), 1-46.

Sánchez Calderón, F. V. (2011). Elementos para una geopolítica de los megaproyectos de infraestructura en América Latina y Colombia.

Asobancaria y Banca de Inversión Bancolombia. (2011). La Infraestructura: Una Alternativa Para la Inversión Privada. En $23^{\circ}$ Simposio Mercado de Capitales, Asobancaria, Medellín, Colombia.

Sánchez Calderón, F. V. (2008). Elementos para una geopolítica de los megaproyectos de infraestructura en América Latina y Colombia. Revista Colombiana de Geografía, (17), 7-21.

Serrano Rodríguez, J. (2010). Financiamiento de infraestructura de transporte. Revista de Ingenierí, (32), 108-116.

Tamayo-Flores, R., \& Hernández-Trillo F. (2006). Financiamiento de la Infraestructura Local en México: Temas Actuales y Perspectivas. Campus Ciudad de México, 1-46.

Villa, J. (2009). Panorama del Financiamiento de Infraestructura en México con Capitales Privados. Experiencia en Estructuración Financiera para el Desarrollo de Infraestructura. Ciudad de México: Fondo Multilateral de Inversiones (FOMIN), del Banco Interamericano de Desarrollo (BID). 